\title{
Associação entre Índices de Disclosure e Características Corporativas das Instituições de Ensino Superior Filantrópicas do Brasil - IESFB
}

\section{Association between Disclosure Index and Corporate Characteristics of Philanthropic Institutions of Higher Education in Brazil - PIHEB}

\author{
Emanoel Marcos Lima ${ }^{a}$; Carlos Alberto Pereira ${ }^{b}$ \\ a Professor da Universidade Federal da Grande Dourados, UFGD; Doutorado em Controladoria e Contabilidade pela \\ Universidade de São Paulo, USP; Dourados, MS - Brasil; E-mail: emanoellima@ufgd.edu.br \\ ${ }^{b}$ Professor da Faculdade de Economia, Administração e Contabilidade, FEA-USP; Doutorado em Controladoria e Contabilidade pela \\ Faculdade de Economia, Administração e Contabilidade - USP, São Paulo, SP-Brasil -E-mail: cap@usp.br
}

\section{Resumo}

O governo brasileiro tem envidado esforços para incrementar o acesso ao ensino superior, provocando o crescimento destas instituições, especialmente daquelas pertencentes ao terceiro setor. Diante de sua relevância para o país, cumpre observar que tais organizações possuem atributos próprios, inclusive no que tange ao seu nível de disclosure. Com base em algumas proposições da Teoria da Divulgação, o objetivo deste artigo é verificar se existe associação entre o nível de disclosure das Instituições de Ensino Superior Filantrópicas do Brasil - IESFB e algumas de suas características corporativas (tamanho, localização, alavancagem, gratuidade, benefícios fiscais e subvenção). O estudo descritivo e quantitativo desenvolve-se pela análise de uma amostra de 146 entidades cadastradas no Conselho Nacional de Assistência Social - CNAS. Para testar as hipóteses estabelecidas foi desenvolvido, com base no estudo de Gordon et al (2002), um modelo de regressão linear múltipla. Como variável dependente foi desenvolvido e utilizado o Índice de Disclosure das instituições de ensino superior filantrópicas do Brasil - IDIESFB, mensurado a partir das características qualitativas de disclosure: (i) visão geral, (ii) desempenho dos serviços, (iii) desempenho financeiro e (iv) situação física e financeira (COY, DIXON e TOWER, 1993). Os resultados dos testes demonstraram associação significativa apenas entre os níveis de disclosure e as características corporativas gratuidade e subvenção, não se observando associação entre esses níveis e as demais variáveis. Adicionalmente, foi possível se observar que a maioria das IESFB pesquisadas apresentou apenas disclosure obrigatório, demonstrando a pouca preocupação dessas instituições com o disclosure voluntário.

Palavras-chave: Instituições de ensino superior; Disclosure; Características corporativas.

\begin{abstract}
The Brazilian government has been making efforts to increase access to higher education, causing the growth of these institutions, especially those belonging to the third sector. Due to its relevance to the country, it must be noted that these organizations have their own attributes, including those related to their level of disclosure. Based on certain propositions of Disclosure Theory, the aim of this paper is to investigate the association between the disclosure level of Philanthropic Institutions of Higher Education in Brazil - PIHEB and some of their corporate characteristics (size, location, leverage, gratuity, tax benefits, and subvention income). The descriptive and quantitative study was developed by analyzing a sample of 146 entities registered with the National Council of Social Assistance- NCSA. For hypothesis testing was developed a multiple linear regression model, based on the study of Gordon et al (2002). As the dependent variable was developed and used the Disclosure Index of Philanthropic Institutions of Higher Education in Brazil - DIPIHEB, measured by the qualitative characteristics of disclosure: (i) overview, (ii) performance of services, (iii) financial performance and (iv) the physical and financial (COY, DIXON and TOWER, 1993). The test results demonstrated a significant association only between the levels of disclosure and corporate characteristics gratuity and subvention income, showing no association between these levels and other variables. Additionally, it was possible to observe that most surveyed PIHEB presented only mandatory disclosure, showing little concern these institutions with voluntary disclosure.
\end{abstract}

Keywords: Higher education institutions;Disclosure; Corporate characteristics. 


\section{INTRODUÇÃO}

O ensino superior no Brasil é sempre um tema que esteve presente nas propostas de todos os governos e não poderia ser diferente, haja vista sua importância para o desenvolvimento do país. De acordo com o art. $3^{\circ}$ da Constituição Federal do Brasil, que estabelece os objetivos fundamentais da República Federativa do Brasil, o Estado deve "[...] erradicar a pobreza e a marginalização e reduzir as desigualdades sociais e regionais." Por sua vez, o art. $6^{\circ}$, que trata dos direitos sociais, define que a educação, a saúde, o trabalho, o lazer, a segurança, a previdência social, a proteção à maternidade e à infância e a assistência aos desamparados constituem direitos sociais dos cidadãos.

Sendo assim, é dever do Estado promover o bem-estar de toda a sociedade sem preconceitos de origem, raça, sexo, cor, idade ou qualquer outra forma de discriminação, atendendo-lhe principalmente nas suas necessidades básicas como: saúde, educação, trabalho e segurança. Segundo a Constituição Federal de 1988, o ensino superior será desenvolvido "com a colaboração da sociedade", no sentido de que esta também possa contribuir para a formação do cidadão. O Estado, ao solicitar a participação da sociedade civil na formação das pessoas, reconhece a sua incapacidade em atender a todas as necessidades educacionais dos indivíduos.

Para auxiliar o Estado, em seu fim institucional, surgiram as Instituições de Ensino Superior Filantrópicas - IESFB. Essas instituições, além da imunidade estabelecida na Constituição Federal, gozam também dos benefícios estabelecidos no art. 55 da Lei Orgânica da Seguridade Social no 8.212, de 24 de julho de 1991. O $\S 7^{\circ}$ do inciso III do art. 195 da CF/88 estabelece que: "São isentas de contribuição para a seguridade social as entidades beneficentes de assistência social que atendam às exigências estabelecidas em lei."

Conforme dados do Ministério da Educação (2008), do total das instituições de ensino superior privadas que funcionavam no Brasil em 2008 (2.483 IES), aproximadamente 90\% eram privadas, sendo que desse total quase $13 \%$ pertenciam à categoria de filantrópicas. As instituições de ensino superior sem finalidades lucrativas, desde que cumpridos os requisitos estabelecidos no Decreto $\mathrm{n}^{0} 2.536$, são consideradas entidades beneficentes de assistência social, portanto isentas das contribuições para a seguridade social de responsabilidade da empresa, sendo que os descontos efetuados dos funcionários, autônomos e empresas devem ser recolhidos normalmente.

Em virtude da renúncia fiscal em favor das IESFB, elas estão obrigadas a prestar contas a determinados órgãos do governo. Entretanto, a sociedade até o ano de 2006 só tinha acesso às demonstrações financeiras dessas entidades através dos jornais. A partir de 2007, o Ministério da Justiça tornou obrigatória, para as instituições que possuem o título de utilidade pública federal, nas quais se incluem as instituições objeto deste estudo, a prestação de contas por meio eletrônico, facilitando o acesso e estando disponível para qualquer pessoa que queira realizar consultas.

O disclosure possui papel essencial na redução da assimetria informacional existente entre a empresa e os stakeholders e é tido como uma das principais formas de comunicação entre a empresa e os diversos usuários interessados em avaliar o desempenho da administração.

Um tema que está sendo abordado pela pesquisa positiva internacional em contabilidade desde a década de 80 é a intitulada "Teoria da Divulgação". Diversos autores têm debatido sobre essa teoria com destaque para os estudos de Verrecchia (1983) e Dye (1985). O principal objetivo dessa teoria é procurar explicar o fenômeno da divulgação de informações financeiras, a partir de algumas perspectivas, buscando entender, entre outros aspectos, as razões econômicas 
para que determinada informação seja voluntariamente divulgada.

Pesquisadores na área de contabilidade, de acordo com Ahmed e Courtis (1999), entre eles: Singhvi e Desai (1971); Buzby (1975); Firth (1979); McNally et al. (1982); Cooke (1989a, 1989b, 1992, 1993); Ahmed e Nicholls (1994); Wallace et al. (1994), e Wallace e Naser (1995), têm estudado a existência de associação entre características empresariais e divulgações dos relatórios anuais corporativos das empresas com finalidades lucrativas, desde 1961, das empresas com finalidades lucrativas. Os achados têm demonstrado que, dentre as características estudadas, o tamanho da empresa e listing status são características que estão associadas significativamente com níveis de divulgação.

Em oposição às investigações ora realizadas, por sua vez, as instituições de ensino superior sem fins lucrativos, talvez em função de suas particularidades, têm recebido por parte dos pesquisadores da área contábil pouca atenção no que diz respeito a estudos que investigam o disclosure por elas realizados. A carência de estudos voltados a esse escopo investigativo atribui à presente investigação potencial contributivo ao incremento de massa crítica a respeito do tema abordado.

Diante da importância do ensino superior para o Brasil, dos constantes esforços do governo federal para que mais pessoas tenham acesso ao ensino superior, e do fato de que as IESFB recebem recursos públicos por meio de renúncia fiscal para o desenvolvimento de suas atividades, é que se estabelece a principal questão de pesquisa que será respondida ao longo deste estudo: Há associação entre características corporativas (tamanho, localização, alavancagem, gratuidade, benefício fiscal e subvenção) e os níveis de disclosure das IESFB? Visando responder a questão de pesquisa formulada, estabelece-se o seguinte objetivo para este trabalho: verificar se existe associação entre características corporativas e nível de disclosure das IESFB.

O presente artigo está dividido em 5 tópicos além deste. A seguir, serão estabelecidas as bases teóricas em que a investigação se apóia, delimitando os conceitos de Instituições de Ensino Superior Filantrópicas, a literatura pertinente com relação ao tema disclosure, bem como evidenciando o estudo de Coy, Dixon e Tower (1993), no qual se baseou o questionário de pesquisa de campo. Além disso, são explicitadas as categorizações metodológicas do estudo, bem como evidenciados os procedimentos para a realização deste. Após a análise dos resultados decorrentes da aplicação do modelo de regressão extraído da relação investigada, são apresentadas as conclusões e referências utilizadas.

\section{REFERENCIAL TEÓRICO-EMPÍRICO}

O marco teórico-empírico de referência aborda conceitos de IESF e os principais estudos relacionados a disclosure, evidenciando as investigações que foram utilizadas como subsídio para a presente investigação.

\subsection{Conceito de instituições de ensino superior filantrópicas}


Essas instituições, por não possuírem finalidades lucrativas, estão classificadas como Terceiro Setor. As IESFB, apesar de pertencerem ao terceiro setor, não se caracteriza $\mathrm{m}$ pela participação voluntária, pois suas atividades são desenvolvidas por funcionários remunerados pela prestação dos serviços, sendo possível, dessa forma, a contratação e remuneração de funcionários para desempenhar as funções necessárias para o desenvolvimento das atividades. Outrossim, em face dos serviços que prestam, exigem a qualificação dos colaboradores, em função não só das exigências do Ministério da Educação, bem como da sociedade.

Para Benedicto (1997, p. 20):

As instituições de ensino - quer particulares, quer públicas, com ou sem fins lucrativos - são organizações constituídas para oferecer benefícios à sociedade. Esses benefícios são de natureza social, cultural, econômica, educativa, tecnológica e moral. Sua existência só tem sentido quando realmente estão contribuindo para o desenvolvimento da comunidade.

A essas características pode-se acrescentar que, diferentemente da maioria das entidades sem finalidades lucrativas, estas entidades sobrevivem com recursos oriundos de mensalidades provenientes da prestação de serviços educacionais e também de subvenções e convênios firmados tanto com a iniciativa privada como com órgãos governamentais.

Observa-se que o lucro não é objetivo dessas entidades, entretanto necessário para a manutenção do patrimônio e para assegurar a continuidade e o cumprimento da missão da instituição. Além disso, adiciona-se às características das entidades do terceiro setor o Certificado de Entidade Beneficente de Assistência Social, expedido pelo Ministério da Educação. O quadro 1 delineia o conceito de IESFB.

Instituições de Ensino Superior com Certificado de Entidade Beneficente de Assistência Social são instituições que apliquem, anualmente, em gratuidades, pelo menos $20 \%$ (vinte por cento) da sua receita bruta total; que promovam a assistência educacional em nível superior, visando à preparação do indivíduo para o mercado de trabalho, provocando, dessa forma, mudanças na sociedade e cujo patrimônio é constituído, mantido e ampliado, a partir de mensalidades, subvenções, doações e convênios com entidades públicas e privadas, não podendo, de forma alguma, seus diretores, conselheiros, sócios, instituidores, benfeitores ou equivalentes receber qualquer remuneração, por qualquer forma ou título, em razão das competências, funções ou atividades que lhes sejam atribuídas pelos respectivos atos constitutivos.

Quadro 1 - Conceito de IESFB

Fonte: Adaptado de Lima (2003, p.45)

\subsection{Literatura sobre Disclosure}

A literatura sobre disclosure esclarece que estudos dessa natureza são realizados sob três perspectivas, segundo Verrecchia (2001). O detalhamento de aspectos fundamentais da teoria de evidenciação e de investigações em que a pesquisa em questão está fundamentada encontram-se nos tópicos a seguir.

\subsubsection{Teoria sobre Disclosure}

Hope (2003) argumenta que disclosure é um fenômeno inerentemente complexo e uma simples teoria pode dar uma explicação parcial. Verrecchia (2001) e Dye (2001) apresentam uma revisão teórica e demonstraram argumentos para a existência de uma teoria unificada ou 
abrangente sobre disclosure.

A taxonomia proposta por Verrecchia (2001, p. 99) engloba três categorias amplas de pesquisa sobre disclosure na contabilidade:

- Pesquisa baseada na associação (association-based disclosure)-busca identificar a associação entre disclosure e o comportamento dos investidores.

- Pesquisa baseada na discricionariedade do disclosure (discretionary-based disclosure) - procura identificar os motivos da divulgação, busca identificar por que os gestores e/ou as empresas decidem divulgar determinadas informações.

- Pesquisa baseada na eficiência do disclosure (efficiency-based disclosure), que debate que tipo de informações são preferidas e mais eficientes, considerando que as informações ainda não foram divulgadas.

Por sua vez, Dye (2001, p. 184) discorda parcialmente de Verrecchia apresentando o seguinte argumento: no que diz respeito ao disclosure obrigatório, concorda que a literatura existente não está suficientemente amadurecida para merecer o status de teoria. Já com relação ao disclosure voluntário, Dye (2001) defende que essa questão pode ser estudada como um apêndice dentro da Teoria dos Jogos, onde a entidade fará ou não disclosure, na proporção em que este seja favorável ou não à entidade.

Dye $(2001,185)$ cita como exemplo uma empresa que, em seu relatório, destaca somente o seu sucesso, programa de redução de custos, e não faz qualquer tipo de citação às suas receitas. De acordo com o autor, nesse caso a teoria permite prever que o crescimento das receitas não foi tão satisfatório como no caso do programa de redução de custos, antes mesmo de examinar a demonstração de resultados. Para Dye (2001), a utilização da premissa de que as empresas ou seus gestores irão divulgar o que acreditam ser favorável à empresa e omitir o que for desfavorável não tem necessariamente ligação com valorização ou desvalorização do preço da ação.

Verrecchia (2001, p. 100) defende que uma teoria abrangente deveria reconhecer todos os aspectos relacionados com o papel da eficiência, dos incentivos e a endogeneidade do processo de mercado, sendo que este abrange as interações entre investidores que são agentes que visam maximizar o seu próprio bem-estar.

Dye (2001) apresenta alguns motivos pelos quais o mercado não consegue pressionar as empresas a fazerem divulgação: (1) desconhecimento por parte dos stakeholders do potencial de informação disponível; (2) interpretação da informação ou da ausência dela de maneira diferenciada pelos investidores; (3) em função do gerenciamento de resultados, a credibilidade do disclosure é comprometida.

De acordo com o modelo analítico proposto por Verrecchia (2001), o disclosure discricionário resulta em cinco hipóteses empiricamente testáveis, as quais têm correlação: (1) positiva com o desempenho da empresa, (2) negativa com os custos de divulgação, (3) positiva com o nível de assimetria informacional entre os investidores externos e a empresa, (4) positiva com a importância dada pela empresa às percepções dos terceiros, e (5) positiva com o nível passado de divulgação.

Verrecchia (2001) e Dye (2001), apesar de algumas discordâncias, concordam que atenção especial deve ser dada para estudos sobre a eficiência do disclosure obrigatório. A discussão entre esses autores sobre as questões relacionadas ao disclosure diz respeito ao mercado de capitais; entretanto, no tasngente ao disclosure das IESFB objeto deste estudo, observa-se que 
ainda se tem muito a avançar, haja vista a escassez de estudos nessa área.

\subsubsection{Associação entre características corporativas e nível de disclosure}

Ahmed e Courtis (1999) desenvolveram estudo com o objetivo de verificar e indicar os fatores de diferenças nos resultados em pesquisas empíricas que buscam identificar a associação entre características corporativas e nível de disclosure. No estudo, os autores aplicaram a técnica de meta-análise em 29 investigações. Os autores citam pesquisa realizada por Cerf's (1961) que foi designado não somente para identificar o maior disclosure, mas para explicar, através da associação com atributos da companhia, porque algumas empresas aderem a melhores políticas de disclosure do que outras.

Ahmed e Courtis (1999, p.37) enfatizam que:

Sucessivos estudos têm investigado a associação entre disclosures e características da empresa empregando uma variedade de índices de disclosure e métodos de pesquisa em diferentes países. Dependendo dos objetivos da pesquisa muitos atributos das empresas têm sido examinados nos estudos de disclosure dos relatórios anuais. As mais freqüentes dessas características das empresas têm sido tamanho, ranking, estrutura de capital (alavancagem), lucratividade e tamanho da empresa de auditoria.(tradução nossa)

Para Ahmed e Courtis (1999), as explicações para selecionar as características das empresas incluem: custos de agência, custos dos proprietários, política de custos, governança corporativa e controle, sinal e assimetria informacional, necessidades de capital, custos judiciais e reputação da empresa de auditoria.

O tamanho da empresa é medido pelo valor contábil dos ativos, valor de mercado da firma, receita total ou número de acionistas e tem sido encontrado por ser significativamente e positivamente associado com níveis de disclosure em vários estudos, conforme citam Ahmed e Courtis (1999), sugerindo que companhias maiores seguem melhores práticas de disclosure nos países desenvolvidos (CERF, 1961; SINGHVI E DESAI, 1971; STANGA, 1974; BUZBY, 1975; BELKAOUI E KAHL, 1978; FIRTH, 1979; COURTIS, 1979; MCNALLY et al., 1982; COOKE, 1989a, 1989b, 1992; WALLACE et al., 1994; INCHAUSTI, 1997) e em desenvolvimento Novos Países Industrializados (NIC) (CHOW e WONG-BOREN, 1987; TAI et al., 1990; AHMED E NICHOLLS, 1994; HOSSAIN et al. 1994; MARSTON E ROBSON, 1997). Ahmed e Courtis (1999) explicam que, apesar de uma consistente associação positiva significante entre tamanho da organização e níveis de disclosure ter sido registrada, nota-se que existe uma ampla variação nos resultados.

Associação positiva também foi verificada com relação à alavancagem, que é medida pelo valor contábil das obrigações e o patrimônio líquido ou pelo valor contábil do ativo total e nível de disclosure (MYERS, 1977; SCHIPPER, 1981; CHOW E WONG-BOREN, 1987; WALLACE et al., 1994, AHMED e COURTIS, 1999).

Jensen e Meckling (1976) argumentam que as firmas com alavancagem mais alta incorrem em mais custos de controle e, sendo assim, elas buscam reduzir esses custos pelo disclosure de mais informações nos relatórios anuais. Em seu estudo, Ahmed e Courtis (1999) afirmam que a evidência empírica relacionada com essa hipótese é inconclusiva, destacando que alguns estudos têm encontrado uma relação significante (COURTIS, 1979; MALONE et al., 
1993; HOSSAIN et al., 1994), enquanto outros não têm encontrado relacionamento (CHOW E WONG-BOREN, 1987; AHMED E NICHOLLS, 1994; WALLADE ET AL., 1994; WALLACE E NASER, 1995; HOSSAIN ET AL., 1995; RAFFOURNIER, 1995).

A rentabilidade da firma também é outra hipótese associada positivamente com o nível de disclosure. Singhvi e Desai (1971) argumentam que maior lucratividade motiva gerenciamento e fornece mais informações porque ela aumenta a confiança dos investidores que, por sua vez, aumenta a compensação do gerenciamento. Sobre essa característica, Ahmed e Courtis (1999) afirmam que evidências empíricas fornecem resultados conflitantes. Uma relação significativamente positiva foi encontrada em alguns estudos (SINGHVI, 1968; SINGHVI E DESAI, 1971; WALLACE et al., 1994), enquanto outros estudos não encontraram tais relacionamentos (MCNALLY et al., 1982; LAU, 1992; RAFFOURNIER, 1995). Uma associação significativamente negativa entre lucratividade e nível de disclosure tem também sido registrada (BELKAOUKI E KAHL, 1978; WALLACE E NASER, 1995).

De acordo com Ahmed e Courtis (1999), vários estudos examinaram a associação entre o tamanho da empresa de auditoria e o nível de disclosure, destacando: Firth (1979), DeAngelo(1981); Wallace et al.(1994), sendo que os resultados dos estudos não são conclusivos. Algumas pesquisas encontraram que empresas de auditoria maiores mostraram uma significativa associação ( $\mathrm{p} \leq 0,05)$ com elevado nível de disclosure (SINGHVI E DESAI, 1971; MALONE et al., 1993; AHMED E NICHOLLS, 1994; HOSSAIN et al., 1994; RAFFOURNIER, 1995; AHMED, 1996; PATTON E ZELENKA, 1997). Resultados similares foram obtidos por outros estudos, mas com menor nível de significância (MACNALLY et al., 1982; TAI et al., 1990). Outros estudos, segundo os autores, não apresentaram relacionamento entre tamanho da firma de auditoria e nível de disclosure (SINGHVI, 1968; COURTIS, 1979; FIRTH, 1979, TONG et al., 1990; WALLACE et al., 1994).

Os autores também analisaram outros estudos que examinaram a associação de disclosure dos relatórios anuais com outras variáveis: Belkaoui e Kahl (1978); Wallace et al.(1994) e Wallace e Naser(1995) incluíram liquidez, sendo que os resultados mostraram relacionamento não significante entre nível de disclosure e a liquidez da firma. O tipo de segmento empresarial também foi investigado por Stanga (1976); Belkaouki e Kahl(1978); McNally et al. (1982); Wallace (1987); Cooke (1989a, 1989b); Wallace et al. (1994). Alguns países apresentaram relacionamento significante (Stanga,1976, nos USA, Belkaoui e Kahl, 1978, no Canadá e Cooke, 1989a e 1989b na Suécia), enquanto outros não (Wallace,1987, na Nigéria, MacNally et al., 1982, na Nova Zelândia e Wallace et al. ,1994, na Espanha).

Outros estudos examinaram o relacionamento do nível de disclosure com a nacionalidade dos gestores, relacionamento com a holding e qualificação do principal executivo contábil na Índia (SINGHVI, 1968); Bangladesh (PARRY E GROVE, 1990; AHMED E NICHOLLS, 1994; AHMED, 1996); Nigéria (WALLACE, 1987); Tanzânia (ABAYO et al., 1990) e Suécia (COOKE, 1989, 1989b). Os resultados encontrados por Singhvi(1968) demonstram que empresas indianas gerenciadas por gestores nacionais evidenciavam menos informações que seus colegas gestores estrangeiros. Abayo et al. (1990) e Ahmed e Nicholls (1994) encontraram que o nível de aceitação com as normas de disclosure obrigatório não teve associação significante com a qualificação profissional pelo principal executivo contábil. Por outro lado, Wallace (1987) e Ahmed e Nicholls (1994) encontraram um relacionamento significante entre nível de disclosure e o relatório da entidade quando a entidade é uma subsidiária de uma companhia multinacional; em oposição a esse resultado Cooke (1989a, 1989b) não encontrou tal relacionamento.

Courtis (1979) desenvolveu estudos com empresas da Nova Zelândia onde examinou 
uma série de variáveis: oportunidade do relatório anual; custo de elaboração do relatório anual, percentual de dividendos; seguro de variação de preços, idade da companhia, taxa de retorno; e emissão de seguros no mercado de ações da Nova Zelândia. Ele encontrou uma associação significativa entre níveis de disclosure em cada uma das variáveis acima, exceto oportunidade e idade da companhia.

\subsubsection{Estudo de Gordon et al. (2002)}

A pesquisa de Gordon et al (2002) buscou analisar os relatórios anuais do período de 1993 e 1994 de 100 instituições de ensino superior dos Estados Unidos, sendo 49 instituições públicas e 51 instituições privadas, com o objetivo de identificar e mensurar fatores associados ao disclosure financeiro. Os relatórios foram analisados utilizando procedimentos de análise de conteúdo para quantificar a extensão dos disclosures, tendo por base os itens de evidenciação apontados no estudo de Engstrom (1988). Os autores utilizaram um sistema de codificação binária, sendo que o item presente recebeu pontuação 1 e o item ausente pontuação 0 . A construção da medida para a extensão do disclosure foi a razão do total de disclosure de uma instituição em relação à pontuação total possível de disclosure. O estudo não levou em consideração a qualidade ou quantidade do disclosure. Os disclosures financeiros examinados foram aqueles encontrados nos relatórios anuais fornecidos pelas instituições, não sendo examinado nenhum outro documento. $\mathrm{Na}$ investigação, os autores utilizaram modelos de regressão de mínimos múltiplos quadrados para testar as 11 (onze) hipóteses, detalhadas abaixo.

Ha1: Faculdades e universidades com elevados níveis de ativos totais divulgam mais informações financeiras do que as outras com menores níveis.

Ha2: Faculdades e universidades com maior número de membros no conselho de administração divulgam mais informações financeiras do que aquelas com menos membros.

Ha3: Faculdades e universidades públicas divulgam mais informações financeiras do que as faculdades e universidades privadas.

Ha4: Faculdades e universidades que são auditadas pelas grandes empresas de auditoria divulgam mais informações financeiras do que as faculdades e universidades auditadas por empresas menores ou empresas que estejam sujeitas a auditoria estadual.

Ha5: Faculdades e universidades altamente endividadas divulgam mais informações financeiras do que as com menores níveis de endividamento.

Ha6: As instituições localizadas em estados com um único conselho de administração divulgam mais informações financeiras do que aquelas instituições com conselho de administração descentralizado, ou seja, em vários estados.

Ha7: Faculdades e universidades privadas cujas operações são proporcionalmente menos dependentes de mensalidades e das receitas de taxas divulgam mais informações do SEA (Service Effort Acomplishment) do que as menos dependentes.

Ha8: Faculdades e universidades privadas com grandes doações divulgam mais informações do SEA do que as com menos doações.

Ha9: Faculdades e universidades privadas que cobram mensalidades e taxas mais elevadas divulgam mais informações do SEA do que as que cobram mensalidades menores. 
Ha10: Faculdades e universidades públicas auditadas por auditores do Estado divulgam mais informações acerca do SEA do que as auditadas pelas empresas de auditoria pública.

Ha11: Faculdades e universidades privadas divulgam informações do SEA mais extensas do que as públicas.

No estudo, as hipóteses $1,7,9,10$ e 11 foram confirmadas, enquanto que as demais hipóteses não foram confirmadas.

\subsubsection{Estudo de Coy, Dixon e Tower (1993)}

Durante o período de 1985 a 1990 ocorreram mudanças consideráveis nos relatórios anuais das instituições de educação superior da Nova Zelândia. O estudo buscou examinar se essas mudanças continuaram, tendo como base o conteúdo do relatório, a oportunidade na publicação e a extensão da distribuição.

No estudo, foram utilizados dois conjuntos de informações. Primeiramente, os relatórios de 1992 publicados até novembro de 1993 são analisados pela qualidade do conteúdo utilizando índice de disclosure desenvolvido por Coy, Tower e Dixon (1993), e comparado com análises similares dos relatórios de 1991 a 1990. Posteriormente, em abril de 1993 foram enviados questionários para 32 instituições com o objetivo de se obter informação de quando e para quem os relatórios anuais de 1992 foram distribuídos.

A oportunidade dos relatórios anuais da educação superior foi considerada sobre dois aspectos: a data quando as demonstrações financeiras são enviadas ao parlamento e a data quando as demonstrações estão disponíveis para usuários externos e o público em geral. A primeira foi chamada pelos autores de oportunidade do processo estatutário e a segunda de oportunidade de accountability pública.

Os autores defendem que os indivíduos têm o direito de examinar os relatórios das instituições a partir da data que os relatórios são disponibilizados ao parlamento, no entanto a oportunidade dos relatórios anuais, no sentido de distribuição ao público é deixada a critério de cada instituição.

Como distribuição os autores consideram o número de relatórios enviados aos indivíduos e outras organizações, o alcance e adequação das pessoas a quem são enviados os relatórios, a sua disponibilidade em bibliotecas, e a forma como a sua acessibilidade é divulgada inclusive os comunicados à imprensa e a utilização de anúncios publicitários. O estudo aponta que a maioria dos relatórios anuais (61\%) é distribuída às pessoas que trabalham na educação superior.

Os relatórios anuais das instituições de educação superior da Nova Zelândia foram avaliados com o auxílio de um índice de disclosure que mede a sua qualidade utilizando uma escala ordinal. Em pesquisa anterior, Dixon et al. (1992) analisaram os relatórios relativos aos anos de 1985 até 1990 utilizando um índice chamado score de disclosure de accountability (ADscore). Este índice incluía 43 itens que os autores identificaram por meio de uma combinação de dedução ou indução e que, na opinião dos autores, contribuem para a accountability pública.

Antes de avaliar os relatórios de 1992, os autores revisaram o método AD-score e decidiram continuar o desenvolvimento do índice, analisando os pontos fracos que se tornaram aparentes, incluindo aqueles atribuíveis à transformação nos relatórios a serem publicados em 
1991 e 1992, em comparação com anos anteriores. Como resultado desta revisão, o número de itens no índice foi reduzido de 43 para 26. Esta redução compreendeu a inclusão de um novo item, o índice de alocação overhead, e a redução dos atuais 43 itens para 25 itens, através da combinação de vários itens que foram previamente tratados separadamente. O índice revisado foi chamado de Score de Disclosure de Accountability Modificado (DAM-score) é, a seguir, apresentado com detalhamento e conteúdo de cada um dos itens.

\section{I - Visão Geral}

\section{1 - Demonstração de Objetivos}

Demonstração que deve incluir os seguintes itens: missão, objetivos e metas de desempenho em específico, concisos, com terminologia compreensível e realista. Os itens devem ter dimensões quantitativas/mensuráveis e fornecer um quadro de tempo.

02 - Relatório descritivo/revisão geral

Relatório emitido pelo presidente do conselho e ou vice-chanceler. Relatório das principais atividades e realizações, no conjunto do contexto social, econômico e política ambiental. Incluindo algumas quantificações com comparativos.

\section{3 - Resumo dos fatos e números}

Principais fatos e números (destaque para dados estatísticos), incluindo tendências e comparativos de 4 anos, possivelmente ilustrações.

\section{4 - Revisão financeira}

Revisão das receitas, custos, ativos, obrigações, projetos de capital e qualquer outro tema financeiro significante. Incluem explicação de tendências, possivelmente ilustrações.

\section{5 - Politicas contábeis}

Detalhamento das políticas contábeis utilizadas e as mudanças ocorridas, evidenciando todos os critérios de apresentação. Razões para mudanças com explicação detalhada e com valor do impacto nas demonstrações.

06 - Informações da diretoria

Endereço, telefone, número do fax, conteúdo da página na internet, data da emissão do relatório anual, membro do conselho e quem ele representa, funcionário sênior da administração.

\section{II - Desempenho dos serviços}

\section{7 - Número de estudantes}

Número de estudantes com informação de pelo menos 4 anos e tendência, discriminando os estudantes em tempo integral e tempo parcial, bem como o nível de estudo: graduação, pósgraduação e outros cursos.

08 - Formandos

Número de formandos por curso com informações de pelo menos 4 anos. 
09 - Emprego/destino dos estudantes da educação

Destino dos estudantes após a formatura, principalmente com relação aos estudantes de anos anteriores.

10 - Publicações

Publicações por curso e tipo (por exemplo, referências de periódicos, artigos, etc.), de pelo menos 4 anos.

\section{1 - Percentual de estudantes na faculdade}

Percentual de estudantes na faculdade em relação ao total de estudantes da instituição.

$12-$ Metas

Descrição clara dos objetivos ou metas especificamente com relação ao serviço de ensino e pesquisa e realizações com relação a cada item. Quantificação quando possível com relação às receitas, processos, custos e resultados. Comparação entre objetivos/metas e realizações.

\section{III - Desempenho financeiro}

\section{3 - Demonstração das Operações - DRE}

Demonstração resumida das operações de pelo menos dois anos para fins de comparação, incluindo notas explicativas e outras informações necessárias para melhor entendimento dos usuários.

\section{4 - Depreciação}

Descrição detalhada da depreciação de todos os ativos em uso, incluindo taxas e vida útil.

\section{5 - Informações orçamentárias}

Todas as principais demonstrações contábeis, incluindo demonstração de operações, fluxo de caixa, custos dos serviços e balanço patrimonial. Mostrando variação e explicação das maiores variações.

\section{6 - Custo unitário por estudante}

Custo unitário por estudante, custo total e por faculdade/escola de pelo menos 4 anos apresentando tendências e ilustrações.

17 - Demonstração do fluxo de caixa

Fluxo de caixa de pelo menos dois anos para fins de comparação, com notas explicativas e outras informações necessárias para que o usuário entenda claramente a reconciliação com superávit ou déficit operacional.

\section{8 - Subsídio para pesquisa}

Informações sobre o valor dos recursos recebidos e destinados para a pesquisa por área de estudo e projeto.

19 - Alocação de overhead 
Composição do total de overheads. Adicionalmente, detalhes da alocação dos overheads para faculdades/escolas, bases de alocação, comparativo da previsão anual.

\section{IV - Situação física e financeira}

\section{0 - Balanço Patrimonial}

Demonstração detalhada, divulgando todos os ativos incluindo terrenos, construções e estoques, e todas as obrigações. Detalhes das reservas e suas modificações. Comparativo anual, notas explicativas extensivas, análise dos ativos por faculdade/departamento. Ativos controlados, mas que legalmente não são próprios, patrimônio financeiro no início do ano corrente, mudanças durante o ano e balanço no final do ano.

\section{1 - Faculdade/Funcionários}

Informações do total de funcionários e por faculdade/escola, classificados em docentes por antiguidade, administrativos, técnicos e outros, a tempo integral e a tempo parcial, com pelo menos informações comparativas de 4 anos. Porcentagem dos docentes com doutorado. Rotatividade do pessoal.

\section{2 - Biblioteca}

Informações financeiras e não-financeiras. Periódicos, quantidades e mudanças do acervo. Detalhes das doações (mecenato). Vídeos e coleções similares. Pessoal e instalações biblioteca. Comparativos.

\section{3 - Investimentos}

Relatório mostrando todos os investimentos, movimentos e resultados. Comparativos, valor de custo e valor de mercado. Base de avaliação.

\section{4 - Compromissos e contingências}

Se não tem compromissos e contingências, demonstrar claramente essa situação. Se existem contingências, separar, divulgando itens individuais com impacto financeiro e afirmando que todos os itens foram divulgados. Se existem compromissos, demonstração com disclosure total, afirmando objetivos/projetos e mostrando o total das despesas esperadas e despesas até a data do encerramento do exercício. Demonstração clara de que todos os itens foram divulgados.

\section{5 - Emprego e informação do patrimônio educacional}

Informações de funcionários e de estudantes, incluindo sexo, etnia, deficiência e idade.

\section{6 - Uso da edificação}

Inclui a área total dos edifícios e instalações no campus, uma indicação de como são utilizados, inclusive para que disciplinas, projetos, laboratórios, etc.

\section{METODOLOGIA DA PESQUISA}

O objetivo deste tópico é apresentar os critérios adotados na pesquisa, em termos de seleção da amostra, estruturação do índice de disclosure e variáveis utilizadas. 


\subsection{Planejamento da pesquisa e coleta de dados}

Uma das formas para verificar a realidade é a pesquisa de campo que, segundo Vergara (2000, p. 47), “[...] é investigação empírica realizada no local onde ocorre ou ocorreu o fenômeno ou que dispõe de elementos para explicá-lo." Segundo Cooper e Schindler (2003), a pesquisa pode ser classificada como descritiva quanto ao objetivo do estudo. Com relação à abordagem do problema, trata-se de uma pesquisa quantitativa, com o emprego de técnica estatística.

A forma selecionada para a coleta dos dados foi por meio de consultas ao banco de dados do Ministério da Justiça e também às páginas da internet das instituições pesquisadas. Foi possível, através das pesquisas realizadas, conhecer um pouco mais da realidade das IESFB inscritas no CNAS e, com base nas informações coletadas, tendo por base o DAM-Score de Coy, Tower e Dixon (1993), elaborar um índice de disclosure das IESFB brasileiras.

\subsection{Variáveis}

Foram definidas, para fins deste estudo, as seguintes variáveis: o tamanho da entidade, alavancagem, localização, o valor das gratuidades, o valor dos benefícios fiscais e o recebimento por parte da entidade de subvenção.

- Tamanho da entidade - definido em função do ativo total da entidade tendo por base os parâmetros definidos pelo Banco Nacional de Desenvolvimento Social - BNDES (2008). conforme abaixo:

a. Micro empresa: até R\$1.200.000,00;

b. Pequena empresa: entre R \$ $1.200 .000,00$ e $\mathrm{R} \$ 10.500 .000,00$

c. Média empresa: entre R\$ 10.500.000,00 e R\$ 60.000.000,00; e

d. Grande empresa: acima de R \$60.000.000,00.

- Alavancagem - medida em função da relação entre exigível total e o ativo total.

- Localização - Região geográfica onde está estabelecida a entidade, sendo que o Brasil está dividido em cinco regiões: norte, nordeste, centro-oeste, sudeste e sul.

- Gratuidade - No caso das entidades objeto deste estudo, na grande maioria, é a prestação de serviços, através de serviço escolar, formação profissional e assistência social à comunidade carente ou a quem dela necessitar. Esses serviços são denominados de beneficências ou gratuidades que são serviços que as IESFB registradas no CNAS estão obrigadas a prestar à comunidade carente como forma de aplicar os recursos recebidos do poder público e também da sociedade, sendo calculado o percentual que representa as gratuidades totais em relação a receita total do exercício de 2007.

- Benefícios Fiscais - significa o quanto a entidade deixou de recolher de tributos aos cofres públicos em função da sua condição de entidade sem fins lucrativos. Nesse estudo foram considerados apenas os valores devidos ao Instituto Nacional do Seguro Social INSS, em função de que essa era uma informação que todas as instituições forneceram. Para fins de aplicação dos testes estatísticos, foi calculado o percentual dos benefícios fiscais em relação a receita total do exercício de 2007. 
- Subvenções - significa se a IESFB recebeu ou não recursos financeiros, materiais ou tecnológicos dos governos federais, estaduais e municipais ou de empresas públicas para aplicação nas atividades, quer seja de custeio quer de capital.

As variáveis foram extraídas do banco de dados do Ministério da Justiça referentes ao exercício de 2007. Sendo assim, o presente trabalho visa testar a existência de evidências estatísticas, ao nível de significância de 5\%, de que algumas características corporativas, previamente definidas, influenciam o nível de disclosure.

\subsection{Hipóteses}

De acordo com Martins e Theóphilo (2007, p.31), “[...] há consenso entre os autores no sentido de que as hipóteses são bem vindas e importantes para os estudos empírico-teóricos". Ainda segundo os autores (2007, p.31), "[...] a formulação e teste de hipótese, no contexto do desenvolvimento de uma pesquisa científica, contribuem para o fortalecimento da consistência dos achados da investigação, junto com os resultados do estudo."

Nesse sentido, buscando contribuir, fortalecer os resultados e verificar o relacionamento entre as variáveis anteriormente estabelecidas, foram estabelecidas as seguintes hipóteses que o presente estudo irá testar.

- Hipótese 1 - O tamanho da instituição está associado ao índice de disclosure das IESFB.

- Hipótese 2 - A alavancagem está associada ao índice de disclosure das IESFB.

- Hipótese 3 - A localização da instituição está associada ao índice de disclosure das IESFB.

- Hipótese 4 - As gratuidades realizadas pelas instituições estão associadas ao índice de disclosure das IESFB.

- Hipótese 5 - Os benefícios fiscais estão associados ao índice de disclosure das IESFB.

- Hipótese 6 - As subvenções estão associadas ao índice de disclosure das IESFB.

Sendo assim, o presente trabalho visa testar a existência de evidências estatísticas, de que algumas características corporativas, previamente definidas, influenciam o nível de disclosure.

\subsection{Construção da variável Disclosure}

Estudos sobre índice de disclosure têm um forte fundamento na literatura contábil. A maioria dos estudos, de acordo com Gordon et al. (2002), tem utilizado uma lista pré-determinada de disclosures que analistas financeiros e/ou stakeholders consideram importantes no processo da decisão de investimento. Os autores têm adotado em suas pesquisas índices ponderados e não ponderados.

A presente investigação focou sobre o disclosure das IESFB brasileiras. Os disclosures examinados foram apenas aqueles encontrados nas informações prestadas pela instituição para 
o Ministério da Justiça e também aqueles disponibilizados em sua página da internet, já que o pedido ao CNAS foi negado. No total foram pesquisadas 146 IESFB.

Nesta pesquisa, optou-se por elaborar um índice de disclosure próprio, a partir do indicador estruturado por Coy, Dixon e Tower (1993), adaptado à realidade brasileira, haja vista não existir nenhum índice no Brasil para as instituições pesquisadas. O índice compreende informações de natureza obrigatória e voluntária, e possibilita a todos os envolvidos, ou seja: governo, gestores e sociedade conhecer: informações de natureza geral, o desempenho dos serviços e financeiros, bem como a situação financeira da instituição.

O índice desenvolvido é composto de 26 questões, sendo que, quando a instituição divulgou o item, recebeu 1, e se não divulgou recebeu 0. Assim como Gordon et al. (2002), não foi realizada qualquer tipo de pontuação em função da quantidade ou da qualidade do disclosure, haja vista que os autores não possuíam informações disponíveis para realizar esse tipo de avaliação. Sendo assim, uma instituição, por exemplo, que divulgou um item referente aos últimos 5 (cinco) anos recebeu a mesma pontuação que a instituição que divulgou a informação apenas de 1 (um) ano. A estrutura utilizada para apuração do Índice de Disclosure das IESFB é o DAM-Score desenvolvido por Coy, Dixon e Tower (1993), que se encontra detalhado no item 2.2.4. $\mathrm{O}$ índice engloba 4 (quatro) conjuntos de informações:

- Informações gerais sobre a instituição (06 itens) - relacionados com os objetivos da instituição, principais fatos e números, políticas contábeis adotadas e informações sobre a diretoria.

- Desempenho dos serviços (06 itens) - relacionados com o desempenho da instituição com relação aos estudantes, formandos, destino dos egressos, publicações, percentual de estudantes e metas.

- Desempenho financeiro (07 itens) - relacionados com o desempenho financeiro da entidade apresentando: resultado das operações, informações detalhadas sobre depreciação e orçamento, custo dos estudantes, fluxo de caixa, subsídio para a pesquisa e alocação de overhead.

- Situação física e financeira (07 itens) - apresenta a situação física e financeira da entidade por meio das seguintes informações: balanço patrimonial, funcionários da faculdade, investimentos, biblioteca, compromissos e contingências, patrimônio educacional e uso da edificação.

Em cada conjunto de informação, a pontuação obtida pela entidade foi apurada pela divisão entre a soma de pontos obtidos e a pontuação total possível do conjunto; o mesmo procedimento foi adotado para apuração do índice de disclosure total.

\subsection{Modelos estatísticos}

$\mathrm{Na}$ investigação em questão foram utilizados modelos de regressão de mínimos múltiplos quadrados para testar as hipóteses acima estabelecidas. De acordo com Hair Jr. et al. (2005, p.32), “[...] regressão múltipla é o modelo de análise apropriado quando o problema de pesquisa envolve uma única variável dependente métrica considerada relacionada a duas ou mais variáveis independentes métricas."

Para fins deste estudo será utilizado como variável dependente o Índice de Disclosure das instituições de ensino superior filantrópicas do Brasil - IDIESFB e como variáveis independentes as seguintes variáveis: Tamanho, Localização, Alavancagem, Gratuidade, Benefícios Fiscais e 
Subvenção. Nesse caso, as variáveis Tamanho, Localização e Subvenção não são variáveis métricas, sendo necessário substituí-las por variáveis dicotômicas, que atuam como variáveis de substituição.

A regressão múltipla permite aos pesquisadores, além de avaliar a importância de cada variável, uma maneira de avaliar a natureza das relações entre as variáveis independentes e dependente. O objetivo, segundo Hair Jr. et al.(2005, p.32), “[...] é prever as mudanças na variável dependente como resposta a mudanças nas variáveis independentes."

Gordon et al. (2002), para testar as hipóteses do seu trabalho, utilizaram os seguintes modelos de regressão:

1) $\mathrm{EXTENT}=\alpha+\beta_{1} \mathrm{LAGASSET}+\beta_{2}$ BRDMBRS $+\beta_{3}$ PUBLPRIV $+\beta_{4} \mathrm{BIG} 6+\beta_{5} \mathrm{DEQUITY}+\varepsilon$

2) $\mathrm{SEA}=\alpha+\beta_{1}$ TUITPCT $+\beta_{2}$ ENDOW $+\beta_{3}$ TUITION $+\varepsilon$

3) $\mathrm{SEA}=\alpha+\beta_{1} \mathrm{CONSBRD}+\beta_{2}$ STATEAUD $+\varepsilon$

4) $\mathrm{SEA}=\alpha+\beta_{1}$ TUITPCT $+\beta_{2}$ ENDOW $+\beta_{3}$ TUITION $+\beta_{4}$ BIG6 $+\beta_{5}$ PUBLPRIV $+\varepsilon$

Modelo 1 - utilizado para testar as hipóteses de 1 a 5 ;

Modelo 2 - utilizado para testar as hipóteses de 7 a 9;

Modelo 3 - utilizado para testar a hipótese 10;

Modelo 4 - utilizado para testar as hipóteses 6 e11.

Onde:

- EXTENT = variável dependente representada pelo índice de disclosure;

- $\mathrm{SEA}=$ variável dependente representada pelo índice de disclosure mensurando somente matrícula e desempenho;

- $\alpha=$ intercepto padrão;

- $\beta=$ coeficiente padrão da regressão;

- LAGASSET = logaritmo natural do ativo total ajustado em função dos recebimentos/ pagamentos;

- BRDMBRS = número de membros no conselho de administração;

- PUBLPRIV = instituição pública ou privada (1=pública, $0=$ =privada);

- BIG6 = BIG6 ou não BIG6 auditor (1=Big6 empresa, 0=Outras);

- DEQUITY = dívida de longo prazo em relação ao patrimônio líquido;

- $\quad$ TUITPCT = anuidades e taxas em relação às receitas correntes totais;

- ENDOW = logaritmo natural do valor justo do fundo de doações;

- $\quad$ TUTION = percentual de anuidades atribuídos para os estudantes em tempo integral da graduação;

- STATEAUD = auditor estatal ou não estatal;

- $\operatorname{CONSBRD}=$ Conselho de administração consolidado ( 1 = sim, 0 = não);

- $\varepsilon=$ padrão normal, termo de erro distribuído randomicamente. 
Os modelos apresentaram os seguintes resultados:

1. Modelo $1-\mathrm{R}^{2}=0,131$, sendo que somente a variável LAGASSET apresentou associação positiva em relação a variável dependente.

2. Modelo $2-\mathrm{R}^{2}=0,321$, sendo que somente as variáveis TUTION e ENDOW apresentaram associação positiva em relação a variável dependente.

3. Modelo $3-\mathrm{R}^{2}=0,028$, tendo a variável STATEAUD apresentado associação positiva em relação a variável dependente.

4. Modelo $4-\mathrm{R}^{2}=0,212$, sendo de somente as variáveis TUITPCT e ENDOW apresentaram associação positiva em relação a variável dependente.

O modelo desenvolvido e utilizado no trabalho dos autores acima citados possui algumas características que diferem substancialmente da realidade brasileira. A seguir são explicitados os ajustes realizados para tal aproximação:

Variável LAGASSET - utilizada para estimar o tamanho da organização e também será utilizada nesse estudo com pequena adaptação. Gordon et al. (2002) enfatizam que talvez a variável explanatória que mais prevalece na extensa literatura de disclosure é o tamanho da organização. Foster (1986, p.44) sugere que a relação positiva que parece existir entre tamanho da empresa e índice de disclosure financeiro pode ser em função de vários fatores subjacentes possíveis, incluindo aumento de custos políticos associados com maiores empresas, menores custos competitivos de disclosures marginais pelas maiores empresas e economias de escala.

Variável BRDMBRS - o número de membros do conselho de administração não é uma informação disponível na base de dados do Ministério da Justiça e também na página da internet das instituições pesquisadas e, sendo assim, não será utilizada nesse estudo.

Variável PUBLPRIV - em 1995, o FASB implementou os SFAS 116 e 117 o que provocou uma significante divergência nos relatórios financeiros anuais das faculdades e universidades públicas e privadas. O estudo realizado por Gordon et al. (2002) foi com base em relatórios realizados antes das mudanças e, sendo assim, passível de comparações, já que tanto instituições públicas como privadas apresentavam os relatórios com os mesmos formatos e orientações. No Brasil as instituições públicas elaboram seus relatórios anuais tendo por base a Lei $n^{\circ} 4.320 / 64$ que estabelece normas e padrões para o setor público. Já as IESFB que são instituições de direito privado estão obrigadas a elaborar seus relatórios com base na Lei $n^{\circ}$ 6.404/76 que é a lei que estabelece normas para as sociedades por ações com algumas alterações estabelecidas por normas brasileiras de contabilidade. Deste modo, também não incluiremos essa variável nesse estudo.

Variável BIG6 - em função da resposta negativa do CNAS em fornecer informações para a essa pesquisa também não foram obtidas informações sobre a empresa de auditoria, apenas sabe-se que todas são auditadas, pois se trata de imposição legal.

Variável DEQUITY - no modelo os autores utilizaram o valor das dívidas em função do patrimônio líquido. Nesse estudo a alavancagem será medida por meio da relação do total das dívidas e ativo total. . Sobre essa questão, Alencar (2007) esclarece que a alavancagem pode ser medida tanto pela relação entre o valor contábil das dívidas e o patrimônio líquido como pelo ativo total.

O modelo utilizado nesta pesquisa para testar a associação entre índice de disclosure e 
algumas características corporativas busca investigar a existência de um relacionamento linear entre as variáveis, e é assim descrito:

\section{IDIESFB $=\alpha+\beta_{1}$ TAMANHO $+\beta_{2}$ ALAVANCAGEM $+\beta_{3}$ LOCALIZAÇÃO $+\beta_{4}$ GRATUIDADE $+\beta_{5}$ BENEFÍCIOFISCAL $+\beta_{6}$ SUBVENÇÃO $+\varepsilon$}

Onde:

- IDIESFB - índice de disclosure das IESFB conforme apresentado anteriormente neste trabalho, sendo transformado posteriormente em logarítmo;

- TAMANHO - conforme descrito no item 3.2.

- ALAVANCAGEM - medida por meio da relação do exigível total e ativo total;

- LOCALIZAÇÃO - região geográfica onde está estabelecida a instituição, sendo utilizada uma variável dicotômica, pois se trata de uma variável não métrica;

- GRATUIDADE - valor aplicado em benefícios à população através de bolsas de estudos e outras formas de assistência social, medido em função do ativo total;

- BENEFÍCIO FISCAL - valor recebido em benefício fiscal, especificamente INSS, medido em função do ativo total;

- SUBVENÇÃO - variável dicotômica, sendo tratada estatisticamente como: (1=recebeu subvenção pública e $0=$ se não recebeu subvenção pública).

Foram incluídas no modelo as variáveis: localização, gratuidade, benefício fiscal e subvenção, não testadas em estudos anteriores relacionados com instituições de ensino superior, mas que, em função das particularidades das instituições em análise, optou-se por incluir no modelo em questão.

\subsection{Tratamento das variáveis}

O processamento estatístico das variáveis foi realizado através do programa estatístico Eviews 4.0, sendo utilizadas, para fins de análise, apenas as observações que tivessem todos os dados válidos. Apesar de constarem na lista 146 (cento e quarenta e seis) instituições, foram consideradas para análise somente 144 (cento e quarenta e quatro) instituições, em virtude de que 2 (duas) instituições não informaram o valor dos seus passivos.

Mediante a aplicação de testes realizados pelas estatísticas t e F, da análise de coeficientes da regressão, coeficientes de determinação da correlação e do erro-padrão buscou-se verificar a confiabilidade das estimativas obtidas. Hair Jr. et.al. (2005, p.160) afirmam que: "testar a significância estatística para os coeficientes estimados em análise de regressão é apropriado e necessário quando a análise é baseada em uma amostra da população e não em um censo." Segundo os autores, éusadoquandoopesquisadornãoestáinteressadona estimativaderegressão apenaspara aquela amostra, mas sim em saber o quanto os resultados são generalizáveis para toda a população.

Neste sentido, de acordo com Hair Jr. et al. (2005, p.160), "os testes de significância dos coeficientes de regressão fornecem uma estimativa estatisticamente baseada na probabilidade de que os coeficientes estimados em muitas amostras de um dado tamanho sejam de fato diferentes de zero." .Os testes de significância de coeficientes de regressão possibilitam ao pesquisador avaliar empiricamente o verdadeiro impacto da sua pesquisa e determina se os impactos representados pelos coeficientes são generalizáveis ou não a toda a população. 


\section{ANÁLISE DOS RESULTADOS}

A tabela a seguir apresenta o índice de disclosure das IESFB do Brasil, tendo por base as informações coletadas no Ministério da Justiça e da página da internet das respectivas instituições.

Tabela 1 - Índice de Disclosure

\begin{tabular}{c|c}
\hline Características qualitativas & Escores \\
\hline I - Visão Geral & 0,37 \\
\hline II - Desempenho dos serviços & 0,02 \\
\hline III - Desempenho financeiro & 0,15 \\
\hline IV - Situação física e financeira & 0,18 \\
\hline Escore médio & 0,18 \\
\hline
\end{tabular}

Fonte: Dados da pesquisa

Observa-se, na Tabela 1, que o grupo que apresenta maior índice é o grupo I com 37\%. Ressalta-se que dois itens que constam desse grupo, demonstração dos objetivos e políticas contábeis, são disclosures obrigatórios exigidos no relatório de prestação de contas do Ministério da Justiça, o que fez com que esse grupo obtivesse um escore maior que os demais grupos.

Primeiramente, executou-se o modelo completo conforme especificado no item 3.5. Posteriormente, em função de que o modelo não cumpriu alguns pressupostos, foram realizados testes para verificar a forma da distribuição dos erros e a ausência de heterocedasticidade, sendo aplicado o teste não paramétrico Jarque-Bera para verificar o comportamento dos erros e o teste White para testar a ausência de heterocedasticidade.

Como não se obteve sucesso, realizou-se alguns procedimentos e testes no sentido de solucionar os problemas. Foram transformadas em logaritmo as seguintes variáveis: índice de disclosure - LNIDIESFB, gratuidade - LNGRAT e benefícios fiscais - LNBF, sendo que, após a transformação, foram executados vários modelos com o objetivo de se alcançar um modelo que fosse significante.

Tabela 2 - Modelo de Regressão

\begin{tabular}{|c|c|c|c|c|}
\hline \multicolumn{5}{|c|}{ Dependent Variable: LNIDIESFB } \\
\hline \multicolumn{5}{|c|}{ Method: Least Squares } \\
\hline \multicolumn{5}{|c|}{ Date: 01/16/09 Time: 12:56 } \\
\hline \multicolumn{5}{|l|}{ Sample: 1146} \\
\hline \multicolumn{5}{|c|}{ Included observations: 146} \\
\hline Variable & Coefficient & Std. Error & t-Statistic & Prob. \\
\hline SUL & -7.227642 & 6.974309 & -1.036324 & 0.3019 \\
\hline SUDESTE & -9.705157 & 6.913567 & -1.403784 & 0.1626 \\
\hline SUBVEN & 5.140164 & 1.604843 & 3.202907 & 0.0017 \\
\hline NORDESTE & -7.916671 & 7.333532 & -1.079517 & 0.2823 \\
\hline MEDIA & 1.038083 & 2.113627 & 0.491138 & 0.6241 \\
\hline LNGRAT & 0.166719 & 0.094393 & 1.766222 & 0.0796 \\
\hline GRANDE & 2.667443 & 1.741318 & 1.531853 & 0.1279 \\
\hline $\mathrm{CO}$ & -2.306382 & 8.061826 & -0.286087 & 0.7752 \\
\hline $\mathrm{C}$ & -105.2333 & 18.93582 & -5.557367 & 0.0000 \\
\hline R-squared & 0.157180 & \multicolumn{2}{|c|}{ Mean dependent var } & -75.63014 \\
\hline Adjusted R-squared & 0.107965 & \multicolumn{2}{|c|}{ S.D. dependent var } & 9.572240 \\
\hline S.E. of regression & 9.040753 & \multicolumn{2}{|c|}{ Akaike info criterion } & 7.301024 \\
\hline Sum squared resid & 11197.73 & \multicolumn{2}{|c|}{ Schwarz criterion } & 7.484945 \\
\hline Log likelihood & -523.9748 & \multicolumn{2}{|c|}{ F-statistic } & 3.193700 \\
\hline Durbin-Watson stat & 2.055263 & \multicolumn{2}{|c|}{ Prob(F-statistic) } & 0.002355 \\
\hline
\end{tabular}

Fonte: Dados da pesquisa. 
Foram retiradas do modelo, conforme Tabela 2, as variáveis Alavancagem e Benefícios Fiscais, sendo que tanto a variável dependente LNIDIESFB e LNGRAT foram transformadas em logaritmo. O modelo mostrou-se significante, apresentando p-value de 0,002355 sendo aprovado, considerando nível de confiança de 1\%. Pelo modelo, as variáveis Subvenção e Gratuidade apresentam evidências estatísticas de que estão associadas à variável dependente, haja vista que o $p$-value encontrado é menor do que o $\alpha$-alfa estabelecido de 0,10 . O poder explicativo do modelo verificado por meio do $\mathrm{R}^{2}$ ajustado é de apenas 0,107965 ou seja o poder explicativo do modelo é de $10,80 \%$.

O modelo apresentado na Tabela 2, não mostrou consistência em relação ao modelo testado por Gordon et.al. (2002) e outros estudos realizados no mercado de capitais no que diz respeito a variável tamanho, já que tanto o estudo de Gordon et al. (2002) quanto os demais estudos já referenciados neste trabalho os resultados comprovam a associação entre o tamanho da empresa e o nível de disclosure, sendo que no presente estudo essa associação não foi comprovada.

Ressalte-se, entretanto, que em relação à variável alavancagem o estudo em questão apresentou consistência em relação à pesquisa de Gordon et al. (2002), não sendo comprovada a associação com o nível de disclosure. Com relação ao poder explicativo do modelo, verificouse aproximação em relação ao resultado encontrado no estudo acima referenciado haja vista que o resultado do $\mathrm{R}^{2}$ ajustado no presente estudo foi de 0,107965 e o do estudo de Gordon et al. (2002) foi de 0,131 .

As demais variáveis testadas na atual investigação não são comparáveis com o estudo de Gordon et al. (2002) e também com outras investigações já que não foram encontrados na literatura estudos sobre disclosure de IESF que possibilitassem a comparação.

\section{CONCLUSÕES}

A associação entre características corporativas e o seu relacionamento com o nível de disclosure em instituições de ensino superior ainda é um assunto pouco explorado internacionalmente. No exterior, existem alguns trabalhos empíricos que orientaram a pesquisa em questão; entretanto, no Brasil talvez motivados pelas dificuldades nas coletas de informações não foram encontrados trabalhos empíricos sobre esse tema.

Os estudos demonstram que a maioria das melhorias que tem ocorrido na accountability das instituições de ensino superior ocorreram devido às mudanças nas legislações no que diz respeito à forma de prestação de contas dessas instituições. Por outro lado, pesquisa realizada com instituições de ensino superior do Canadá por Nelson, Banks e Fisher (2003) indicou que mudanças na administração, a percepção de necessidade em comunicar-se com os stakeholders ou a necessidade de um sistema de informação interna melhor foram os fatores que influenciaram as melhorias, sendo dessa maneira avanços voluntários e não obrigatórios.

Nesse aspecto, no Brasil infelizmente as mudanças na legislação para as IESFB não fortalecem a accountability pública, ocorrendo dessa forma um retrocesso com relação a essa questão. Embora se discuta muito sobre a importância de informações não financeiras para organizações sem fins lucrativos, elas ainda não são exigidas. 
Algumas poucas IESFB têm disponibilizado em suas páginas na internet além das demonstrações contábeis anuais, o relatório da administração, o parecer da auditoria e o balanço social. Essa atitude por parte dessas instituições demonstra a preocupação em relação à accountability pública a partir do momento em que além de fornecer informações aos órgãos reguladores coloca também a disposição dos diversos stakeholders para que realizem as suas análises.

Os resultados desse estudo mostram que a maioria das IESFB pesquisadas só apresenta disclosure obrigatório, demonstrando a pouca preocupação das instituições com o disclosure voluntário. O DAM-score, desenvolvido por Coy, Dixon e Tower (1993) além de avaliar as divulgações financeiras e não financeiras das instituições de ensino superior provocou em universidades da Nova Zelândia uma busca pela melhoria dos seus índices.

A fim de se verificar a existência ou não de associação entre as características corporativas e o nível de disclosure das instituições de ensino superior pesquisadas, utilizou-se um modelo de regressão linear desenvolvido com base no estudo de Gordon et al (2002). Constatou-se, por meio dos resultados da aplicação desse modelo, que existe relação apenas entre os índices de disclosure e as características corporativas "gratuidade" e "subvenção", não se observando associação entre esses índices e as demais variáveis (tamanho, localização, benefícios fiscais e alavancagem). Importa salientar que os resultados encontrados são incomparáveis com os outros estudos no que diz respeito ao poder de explicação do modelo utilizado. Verificou-se também a aproximação ao modelo 1 testado no estudo de Gordon et al (2002)

Cabe destacar que a associação entre níveis de divulgação e características corporativas em instituições de ensino ainda é um tema pouco explorado na literatura nacional e internacional. No exterior, existem alguns trabalhos empíricos que orientaram esta pesquisa. Entretanto, no Brasil, talvez pelas dificuldades na coleta de informações que dêem suporte a esse tipo de pesquisa, não foram encontrados trabalhos empíricos sobre esse tema.

Com esse estudo, pode-se constatar a necessidade e possibilidade de ampliação desta pesquisa, especialmente, no que diz respeito à necessidade de se:

1. entender os fatores que levam a maioria das IESFB a realizar apenas disclosure obrigatório;

2. analisar e investigar outras variáveis que podem contribuir para a evidenciação voluntária;

3. ampliar o período de investigação, com o objetivo de verificar o comportamento das variáveis ao longo do tempo; e

4. investigar, por meio de pesquisas junto aos dirigentes das IESFB que tomam decisões as razões para divulgação ou retenção de informações. 


\section{REFERÊNCIAS}

ABAYO, A.G.; ADAMS, C.A.\& ROBERTS, C.P. Measuring the quality of corporate disclosure in less developed countries with particular reference to Tanzania. Departament of Accounting and Finance, University of Glasgow, UK, 1990.

AHMED, Kamran. Disclosure policy choice and corporate characteristics: a study of Bangladesh. Asia-Pacific Journal of Accounting, Vol. 3, n.01, p.183-203, 1996.

AHMED, K.; NICHOLLS, D. The effect of non-financial company characteristics on mandatory disclosure compliance in developing countries: the case of Bangladesh. The International Journal of Accounting, Vol. 29, n.01, p.62-77, 1994.

AHMED, Kamran; COURTIS, Jonh K. Association between corporate characteristics and disclosure levels in annual reports: a meta analysis. British Accounting Review, Kindlinton n.31, p.35-61, 1999.

ALENCAR, Roberta Carvalho de. Nível de disclosure e custo de capital próprio no mercado brasileiro. 2007, 104 f. Tese (Doutorado) - Universidade de São Paulo.

BELKAOUI, A. \& KAHL, A. Corporate Financial Disclosure in Canada, Research Monograph, Canadian Certified General Accountants Association Vancouver n. 1:, 1978.

BENEDICTO, Gideon Carvalho de. Contribuição ao estudo de um sistema de contabilidade gerencial para uma gestão eficaz das instituições de ensino. São Paulo, 1997. 230 p. Tese (Doutorado em Controladoria e Contabilidade) - Faculdade de Economia, Administração e Contabilidade da Universidade de São Paulo.

BNDES. Dados a respeito de porte das organizações. Disponível em: www.bndes.gov.br/ conhecimento/cartilha/cartilha.pdf . Acesso em 10.09.2008,

BRASIL. Constituição de 1988: Constituição Federal. Organizador: Pedro de Milanélo Piovezane; Coordenadora: Dulce Eugênia de Oliveira. 4. ed. São Paulo: Rideel, 1999.

Decreto n. 2.536 de 06/04/1998. Dispõe sobre a concessão do Certificado de Entidade de Fins Filantrópicos a que se refere o inciso IV do art. 18 da Lei n. 8.742, de 7/12/1993, e dá outras providências. Disponível em: www.senado.gov.br. Acesso em: 15/08/2004.

. Lei n. 8.212, de 24/07/1991. Dispõe sobre a organização da Seguridade Social, institui Plano de Custeio e dá outras providências. Disponível em: http://wwwt.senado.gov.br/servlets/ NJUR.Filtro?tipo=LEI\&secao=NJUILEGBRAS\&numLe. Acesso em: 15/08/2004.

Lei n. 6.404, de 15/12/1976. Dispõe sobre as Sociedades por Ações. Disponível em: $<$ http://www81.dataprev.gov.br/sislex/paginas/42/1976/6404.htm>. Acesso em: 11/02/2008.

Lei n. 4.320, de 17/03/1964. Estatui Normas Gerais de Direito Financeiro para elaboração e contrôle dos orçamentos e balanços da União, dos Estados, dos Municípios e do Distrito Federal. Disponível em: http://www.planalto.gov.br/ccivil_03/Leis/L4320.htm>. Acesso em: 11/02/2008.

CHOW, C.W. \& WONG-BOREN, A. . Voluntary financial disclosure by mexican corporations. The accounting review, Vol. 62, n. 3, july, p. 533-541, 1987.

COOKE, T.E. Disclosure in the corporate annual report of swedish companies. Accounting and Business Research, Vol. 19, spring, p. 113-122, 1989a. 
Voluntary corporate disclosure by swedish companies. Journal of International Financial Management And Accounting, Vol. 1, n. 2, p. 171-195, $1989 \mathrm{~b}$.

COOPER, D.R. \& SCHINDLER, P.S. Métodos de Pesquisa em Administração. 7. ed. Porto Alegre: Bookman, 2003.

COY, D.; TOWER, G.; DIXON, K. Quantifying the quality of tertiary education annual reports. Accounting and Finance, [S.1.], v.33, p.121-129, november 1993.

Courtis, J.K. Annual Report Disclosure in New Zealand: Analysis of Selected Corporate Attributes. Research Study 8, University of New England, Armidale, Australia, 1979.

DEANGELO,L.E. Auditor Size and Audit Quality. Journal of Accounting and Economics, Vol. 3, n. 4, p. 183-199, 1981.

DIXON, Keith, COY, David, TOWER, Greg. External reporting by New Zealand universities 1985-1989: improving accountability. Financial Accountability and Management. 7(3), Autumm, p. 159-178, 1992

DYE, Ronald A. Disclosure of non proprietary information. Journal of Accounting Research, v.23, n.1, p. 123-145, Spring, 1985.

An evaluation of "essays on disclosure" and the disclosure literature in accounting. Journal of Accounting and Economics. Amsterdam: v.32, n.1-3, p.181-235, 2001.

ENGSTROM, J.H. Information needs of college and university decision makers, Research Report,Governmental. Accounting Standards Board, Norwalk, CT, 1988.

FIRTH, M. The E $\square$ ect of Size, Stock Market Listings, and Auditors on Voluntary Disclosure in Corporate Annual Reports. Accounting and Business Research, Vol. 9, n. 36, Autumn, p. 273-280, 1979.

INEP. Dados das instituições de ensino superior no Brasil. Disponível em www.inep.gov.br. Acesso em 10/11/2008.

FOSTER, G. Financial statement analysis. NJ: Prentice-Hall, Englewood Cliffs, 1986.

GORDON, T.; FISCHER, Mary; MALONE, David; TOWER, Greg. A comparative empirical examination of extent of disclosure by private and public colleges and universities in the United States. Journal of Accounting and Public Policy, [S.1.], 21, p. 235-275, 2002.

HAIR JR., Joseph F. et al. Análise multivariada de dados. Porto Alegre: Bookman, 2005.

HOPE, Kristian-Ole. Firm-level Disclosures and the Relative Roles of Culture and Legal Origin. Journal of International Financial Managerial and Accounting, [S.1.], v.14, n.3, p. 218-248, 2003.

HOSSAIN,M.,TAN,L.M.\& ADAMS,M.Voluntary Disclosure in an Emerging Capital

Market: Some Empirical Evidence from Companies Listed on the Kuala Lumpur Stock Exchange. The International Journal of Accounting, Vol. 29, n. 3, p. 334-351, 1994.

JENSEN, Michael C.; MECKLING, William H. Theory of the firm: managerial behavior, agency costs and ownership structure. Journal of Financial Economics, Amsterdam, v.3, n.4, p. 305-360, oct. 1976 .

LAU, A. Voluntary Financial Disclosure By Hong Kong Listed Companies. Hong kong manager, may/june, p. 10-19, 1992. 
LIMA, Emanoel Marcos. Contribuição para apuração e evidenciação dos resultados das instituições de ensino superior com certificado de entidade beneficente de assistência social - IESCEBAS. São Paulo, 2003. 183 p. Dissertação (Mestrado). Faculdade de Economia, Administração e Contabilidade da Universidade de São Paulo.

MALONE, D., FRIES, C. \& JONES, T. An Empirical Investigation of the Extent of

Corporate Financial Disclosure in the Oiland Gas Industry. Journal of Accounting, Auditing and Finance, vol. 8, no. 3, summer, p. 249-273, 1993.

MARTINS, Gilberto de Andrade; THEÓPHILO, Carlos Renato. Metodologia da investigação científica para ciências sociais aplicadas. São Paulo: Atlas, 2007.

MCNALLY, G.M., ENG, L.H. \& HASSELDINE, C.R. Corporate Financial Reporting in New Zealand: An Analysis of User Preference, Corporate Characteristics and Disclosure Practices for Discretionary Information. Accounting and Business Research, vol.13,winter,pp. 11-20, 1982.

NELSON, Morton; BANKS, William; FISHER, James. Improved accountability disclosures by Canadian universities. Canadian Accounting Perspectives, [S.1.], 2, 1; ABI/INFORM Global, 2003.

PARRY, M.J. \& GROVE, R.E. Does Training More Accountants Raise the Standard of Accounting in Third World Countries. Research in Accounting in Emerging Economies, vol. 1, jai press, London, p. 3-54, 1990.

PATTON, J. \& ZELENKA, I. An Empirical Analysis of the Determinants of the Extent of Disclosure in Annual Reports of Joint Stock Companies in the Czech Republic. The European Accounting Review, vol. 6, n. 4, p. 605-626, 1997.

RA $\square$ OURNIER,B.The Determinant sof Voluntary Financial Disclosure by Swiss Listed Companies. The European Accounting Review, vol. 4, n. 2, pp. 261-280, 1995.

SINGHVI, S. Characteristics and Implications of Inadequate Disclosure: A Case Study of India. The International Journal of Accounting, vol. 3, n. 2, spring, pp. 29-43, 1968.

SINGHVI, S.S. \& DESAI, H.B. An Empirical Analysis of the Quality of the Corporate Financial Disclosure. The Accounting Review, vol. 46, n. 1, january, p. 120-138, 1971.

STANGA, K.G. Disclosure in Published Annual Reports. Financial Management, winter, p. 42-52, 1976.

TAI, B.Y.K., AU-YEUNG, P.K., KWOK, M.C.M. \& LAU, L.W.C. Non-Compliance with Disclosure Requirements in Financial Statements: The Case of Hong Kong Companies.The International Journal of Accounting, vol. 25, n. 2, pp. 99-112, 1990.

TONG,L.T.,KIDAM,Z.A. \& CHEONG,P.W .Information Needs of Users and Voluntary Disclosure Practices of Malaysian Listed Corporations. Malaysian Accountant, April, pp. 2-6, 1990.

WALLACE, R.S.O. Disclosure of Accounting information in Developing Countries: A Case Study of Nigeria. Ph. D. Thesis, university of exeter, UK, 1987.

WALLACE, R.S.O., NASER, K. \& MORA, A. The Relationship Between Comprehensiveness of Corporate Annual Reports and Firm Characteristics in Spain. Accounting and Business Research, vol. 25, n. 97, winter, pp. 41-53, 1994.

WALLACE, R.S.O. \& NASER, K. Firm-Specific Determinants of Comprehensiveness of 
Mandatory Disclosure in the Corporate Annual Reports of Firms on the Stock Exchange of Hong Kong. Journal of Accounting and Public Policy, Vol. 14, pp. 311-368, 1995.

VERGARA, Sylvia Constant. Projetos e relatórios de pesquisa em administração. 3. ed. São Paulo: Atlas, 2000.

VERRECCHIA, Robert E. Discretionary disclosure. Journal of Accounting and Economics, n. 5, p. 179-194, 1983.

Essays on disclosure. Journal of Accounting and Economics, Amsterdam, v.32, n.1-3, p.97-180, 2001. 


\section{ENDEREÇO DOS AUTORES:}

\section{Emanoel Marcos Lima}

Universidade Federal da Grande Dourados, FACE.

Rodovia Dourados-Itahum, Km 12

Dourados, MS - Brasil

79800-000

\section{Carlos Alberto Pereira}

Universidade de São Paulo, Faculdade de Economia Administração e Contabilidade, Departamento de Contabilidade Atuária.

Rua Professor Luciano Gualberto, 908, Cidade Universitária.

São Paulo, SP - Brasil

05508-900 